\title{
Sulfated modification, characterization and monosaccharide composition analysis of Undaria pinnatifida polysaccharides and anti-tumor activity
}

\author{
FU-LING WANG ${ }^{1,2}$, YU-BIN JI ${ }^{1,2}$ and BO YANG ${ }^{1,2}$ \\ ${ }^{1}$ School of Pharmacy, Engineering Research Center for Medicine, Harbin University of Commerce; \\ ${ }^{2}$ Engineering Research Center of Natural Anticancer Drugs, Ministry of Education, Harbin, Heilongjiang 150076, P.R. China
}

Received September 21, 2018; Accepted December 19, 2019

DOI: $10.3892 /$ etm. 2020.8720

\begin{abstract}
Undaria pinnatifida (U. pinnatifida) polysaccharides (UPPS) are considered to be the major bioactive components of $U$. pinnatifida. The aim of the present study was to investigate the separation, sulfated modification, characterization and monosaccharide composition of UPPS. The optimal processing conditions were as follows: Distilled water-to-solid ratio, $50 \mathrm{ml} / \mathrm{g}$; extraction time, $300 \mathrm{~min}$; and extraction temperature, $90^{\circ} \mathrm{C}$. The major polysaccharide fraction of $U$.pinnatifida (UPPS-B1) was purified via DEAE-52 and Sephadex G-200 column chromatography. The chlorosulfonic acid-pyridine method was applied for sulfation modification. UPPS-B1 and sulfated (S)-UPPS-B1 were characterized via chemical analysis, ultraviolet-visible and Fourier-transformed infrared spectroscopy, gas chromatography and high-performance liquid chromatography. The total sugar content of UPPS-B1 and S-UPPS-B1 was 79.78 and $77.28 \%$, respectively. The sulfate radical content of UPPS-B1 and S-UPPS-B1 was 8.53 and $29.12 \%$, whilst the content of uronic acid was 9.29 and $7.98 \%$, respectively. The average molecular weight of UPPS-B1 and S-UPPS-B1 was determined to be 37 and $110 \mathrm{kD}$, respectively. UPPS-B1 was considered to be a heteropolysaccharide composed of xylose, mannose, glucose and galactose at a ratio of 7.9:8.7:12.0:9.8. In addition, S-UPPS-B1 was a heteropolysaccharide composed of xylose, mannose, glucose and galactose at a ratio of 1.0:9.7:6.4:1.6. The results of the tumor growth inhibition experiment demonstrated that UPPS-B1 exhibited anti-tumor activity in vivo, which was improved following sulfation to yield S-UPPS-B1.
\end{abstract}

Correspondence to: Professor Yu-Bin Ji, School of Pharmacy, Engineering Research Center for Medicine, Harbin University of Commerce, 138 Tongda Street, Daoli, Harbin, Heilongjiang 150076, P.R. China

E-mail: 524640082@qq.com

Key words: Undaria pinnatifida, polysaccharides, sulfated modification, characterization, monosaccharide composition, antitumor activity

\section{Introduction}

At present, a wide variety of drugs are commercially available for the treatment of cancer. However, the majority of these have serious adverse effects. Polysaccharides are rich in sulfate radicals and have been regarded as one of the most effective compounds for cancer prevention, constituting the major natural resource of effective novel anti-cancer agents (1).

Undaria pinnatifida (U. pinnatifida), also known as Qundaicai in China, has long been used as a traditional Chinese medicine and functional food source in countries including China, Korea, Australia, New Zealand and Japan. It contains a variety of nutrients, including polysaccharides, proteins and minerals (2). Undaria pinnatifida polysaccharides (UPPS) exert multiple biological functions, including immunomodulatory, anti-cancer, anti-oxidation and anti-viral activities (3).

Previous studies have demonstrated that the anti-tumor activities of Undaria pinnatifida are closely associated with it being rich in sulfated polysaccharides (4). The sulfation of polysaccharides not only enhances the water solubility of such compounds but also improves certain biological activities (5), including anti-viral, immunostimulant, anti-oxidant and anti-tumor effects (6). The chlorosulfonic acid-pyridine method is widely used to produce highly active and functional polysaccharide derivatives, producing high yields and a degree of product substitution (7).

Few studies have characterized and determined the monosaccharide composition of UPPS. In the present study, UPPS were extracted, isolated, purified and modified, and the structural differences between UPPS and sulfated (S)UPPS were subsequently analyzed using DEAE-52 cellulose column chromatography, Sephadex G-200 column chromatography and instrumental analyses. The anti-tumor activity of UPPS and its sulfated derivatives were then evaluated.

\section{Materials and methods}

Chemicals. The dried whole plant of $U$. pinnatifida was purchased from a store (Dalian Longchang Aquatic Products Co., Ltd.) in Dalian, China. The material was identified by the corresponding author. DEAE-52 cellulose and 
Sephadex G-200 were purchased from Whatman Co., Ltd. Monosaccharide standards, T-series dextrans were purchased from Sigma-Aldrich (Merck KGaA). All other reagents used were of analytical grade. The murine sarcoma S180 cell line (original CCRF S-180 II) was obtained from Cobioer Biosciences Co., Ltd. (Nanjing, China) and were cultured in RPMI 1640 medium containing 10\% FBS (Hangzhou Sijiqing Biological Engineering Materials Co., Ltd.) and incubated at $37^{\circ} \mathrm{C}$ in an atmosphere containing $5 \% \mathrm{CO}_{2}$. Kunming mice (POO101009) were provided by the Animal Experiment Centre of Heilongjiang University of Chinese Medicine. They were kept in a temperature- and humidity-controlled environment under a $12 \mathrm{~h}$ light/dark cycle and provided with food and water ad libitum. The experimental protocol was approved by the Institutional Animal Care and Use Committee of Heilongjiang University of Chinese Medicine (Harbin, China).

Extraction and isolation of polysaccharides. Dry U. pinnatifida was smashed using a high-speed disintegrator. The resulting powder was refluxed with $95 \%$ ethanol at $80^{\circ} \mathrm{C}$ for $2 \mathrm{~h}$ to remove any lipids and colored materials. The residue was air-dried to obtain pre-treated powder. The powder $(100 \mathrm{~g})$ was extracted using $5 \times 10^{3} \mathrm{ml}$ distilled water at $90^{\circ} \mathrm{C}$ for $5 \mathrm{~h}$. Following centrifugation at $1,157 \mathrm{x}$ g (room temperature) for $10 \mathrm{~min}$, the supernatant was collected. The extracts were then concentrated using a vacuum rotary evaporator and precipitated with ethanol to a ethanol concentration of $80 \%$. The mixture was stirred and kept overnight at $4^{\circ} \mathrm{C}$ to precipitate the polysaccharides and the resulting precipitate was collected via vacuum filtration. The polysaccharides were then sequentially washed with anhydrous ethanol, acetone and petroleum ether. The precipitates were freeze-dried to obtain crude polysaccharides.

Crude polysaccharide powder $(5 \mathrm{~g})$ was dissolved in $300 \mathrm{ml}$ distilled water and the resulting polysaccharide solution was subsequently deproteinized using the Papain method. The deproteinized polysaccharide aqueous solution was adjusted to a $\mathrm{pH}$ of 8-9 using ammonia solution, $~ 10 \%$ $\mathrm{H}_{2} \mathrm{O}_{2}$ was added and the solution was incubated overnight at $4^{\circ} \mathrm{C}$. Samples were then dialyzed against tap water for $72 \mathrm{~h}$ and distilled water for $24 \mathrm{~h}$ [dialysis bag; cut-off molecular weight (Mw), 8,000-14,000 Da]. The solution was concentrated using a vacuum rotary evaporator and submitted to precipitate with four times the volume of pure ethanol. The resultant mixture was stirred and kept overnight at $4^{\circ} \mathrm{C}$ and subsequently collected via vacuum filtration. Polysaccharides were then sequentially washed with anhydrous ethanol and acetone, and freeze-dried to produce semi-pure polysaccharides.

Purification of the major polysaccharide. The crude polysaccharide mix (300 mg) was dissolved in $60 \mathrm{ml}$ distilled water and then injected into an anion exchange chromatography column $(2.6 \times 55 \mathrm{~cm})$ of DEAE-52 cellulose $(140 \mathrm{~g})$ equilibrated with three column volumes with distilled water. After sample loading, the column was sequentially eluted with distilled water and $\mathrm{NaCl}$ aqueous solution $(0.1,0.2,0.3,0.4,0.5$ and $0.8 \mathrm{M})$ at a flow rate of $1 \mathrm{ml} / \mathrm{min}(10 \mathrm{~min} /$ tube $)$. A total of $3,000 \mathrm{ml}$ $\mathrm{NaCl}$ eluate was collected. The major polysaccharide fractions were obtained using a fraction collector and identified with the phenol-sulfuric acid method and then combined. Samples were then dialyzed. The dialyzed solution was concentrated and freeze-dried (8).

The polysaccharide fractions $(10 \mathrm{mg})$ were dissolved in $2 \mathrm{ml}$ distilled water and injected into a column $(1.6 \times 80 \mathrm{~cm})$ of Sephadex G-200 (10 g) equilibrated with distilled water. After sample loading, the column was eluted with $\mathrm{NaCl}$ aqueous solution $(0.05 \mathrm{M})$ at $0.1 \mathrm{ml} / \mathrm{min}(20 \mathrm{~min} /$ tube $)$ for a total of $160 \mathrm{ml}$. The major polysaccharide fractions were collected using a fraction collector and dialyzed. The dialyzed solution was concentrated and freeze-dried.

Purity determination. The purity of the polysaccharides was determined via high-performance liquid chromatography (HPLC; 2695 system; Waters) with a gel-filtration chromatographic column containing Ultrahydrogel ${ }^{\mathrm{TM}}$ Linear. A refractive index detector (RID) was also utilized (2414 system; Waters). The purified polysaccharide (2 $\mathrm{mg}$ ) was dissolved in distilled water $(10 \mathrm{ml})$ and passed through a filter (pore size, $0.22 \mu \mathrm{m})$. The column was maintained at a temperature of $40^{\circ} \mathrm{C}$, eluted with distilled water at a flow rate of $0.8 \mathrm{ml} / \mathrm{min}$ and detection was performed using RID.

Chemical analysis. The content of total sugar was measured using the phenol-sulfuric acid method (9) with D-glucose utilized as the standard. The content of total sugar was calculated as follows: Polysaccharide content $(\%)=[\mathrm{X} x$ volume $\mathrm{x}$ dilution ratio/amount of polysaccharide $(\mathrm{g})] \mathrm{x} 100$, where $\mathrm{X}$ is the absorbance calculated according to the regression equation of the standard curve.

The content of uronic acid was measured via the sulfuric acid-carbazole method, adopting D-glucuronic acid as the standard (10).

The content of sulfate radicals was measured using the gelatin-barium chloride method (11). The degree of sulfate substitution (DS) was calculated as follows: $\mathrm{DS}=(1.62 \times \mathrm{S} \%) /(32-1.02 \times \mathrm{S} \%)$, where $\mathrm{S}$ is the content of sulfur within the sample.

Sulfated modifications. Pyridine $(60 \mathrm{ml})$ was added to a three-necked flask equipped with a condenser and stirrer and cooled in an ice bath. Chlorosulfonic acid $(10 \mathrm{ml})$ was slowly dripped into the pyridine solution for $\sim 40 \mathrm{~min}$. Samples were removed from the ice bath after a large quantity of yellow solid product had appeared in the flask. UPPS fraction B1 (UPPS-B1) (100 mg) was suspended in dimethylformamide $(10 \mathrm{ml})$ and agitated with a magnetic stirrer for $20 \mathrm{~min}$, after which the mixture was added to the flask. The flask was then immediately placed in a pre-heated water bath $\left(85^{\circ} \mathrm{C}\right)$. The reaction was stirred for $3 \mathrm{~h}$ under $85^{\circ} \mathrm{C}$. The flask was then cooled to room temperature and ice water was slowly added. The solution was then neutralized with saturated $\mathrm{NaOH}$ and a triploid volume of pure ethanol was added. The precipitate was collected via centrifugation $(1,157 \mathrm{x} \mathrm{g}$ for $10 \mathrm{~min}$, room temperature), dissolved in water and dialyzed with distilled water for 3 days. The solution was concentrated and freeze-dried to obtain the sulfated derivative, S-UPPS-B1.

Ultraviolet $(U V)$ spectroscopy. UV spectroscopy is regularly used to detect whether proteins or nucleic acids are present in polysaccharides (12). A total of $1 \mathrm{mg} / \mathrm{ml}$ UPPS-B1 and 
S-UPPS-B1 were each prepared using deionized water. Subsequently, a UV spectrum scan was performed using a DB-20 UV visible spectrophotometer (Dynamica Pty Ltd.) in the range of $200-400 \mathrm{~nm}$.

Fourier-transform infrared (IR) spectroscopy. Fourier-transform IR spectroscopic analysis is utilized to identify the fundamental groups of polysaccharide structures (13). IR analysis was performed using a Fourier transform IR spectrophotometer (FTIR-8001; Shimadzu Corp.). A total of $2 \mathrm{mg}$ of each polysaccharide sample and $400 \mathrm{mg}$ of $\mathrm{KBr}$ were mixed, ground for 5-10 min with an agate mortar and pressed into a $1-\mathrm{mm}$ tablet (14). IR spectra were recorded in the frequency range of 4,000-400 $\mathrm{cm}^{-1}$.

Monosaccharide composition analysis. It is widely recognized that the monosaccharide composition of natural polysaccharides is closely associated with its bioactivity (15). The hydrolyzed and acetylated derivatives of the polysaccharides assessed were loaded onto a gas chromatography-mass spectrometer (GC-MS; model 6890/5973N-GC/MSD; Agilent Technologies, Inc.). Each sample (10 mg) was hydrolyzed using $3.0 \mathrm{ml}$ of $2 \mathrm{M}$ trifluoroacetic acid at $100^{\circ} \mathrm{C}$ for $5 \mathrm{~h}$ in a sealed tube. The hydrolysate was dried with a nitrogen blowing instrument and further dried in a desiccator filled with $\mathrm{P}_{2} \mathrm{O}_{5}$ for $24 \mathrm{~h}$. For GC-MS analysis, the complete hydrolysate was mixed with $1.0 \mathrm{ml}$ pyridine and fully dissolved. A total of $0.4 \mathrm{ml}$ hexamethyldisilazane and $0.2 \mathrm{ml}$ trimethylchlorosilane was then added and the mixture was shaken at room temperature for $5 \mathrm{~min}$. Following centrifugation at $9,391 \mathrm{~g}\left(4^{\circ} \mathrm{C}\right)$ for $30 \mathrm{~min}$, the supernatant was analyzed via GC-MS. The quantity of the monosaccharide component was determined via the peak area. The total injection volume was $1 \mu \mathrm{l}$. GC conditions included the use of an HP-5MS quartz capillary column (0.25 mm x30 m; Agilent Technologies, Inc), an inlet temperature of $270^{\circ} \mathrm{C}$ and a temperature program of $150^{\circ} \mathrm{C}$ for $1 \mathrm{~min}$, a temperature increase by $10^{\circ} \mathrm{C} / \mathrm{min}$ to $250^{\circ} \mathrm{C}$ and a hold at $7 \mathrm{~min}$. The MS conditions were an interface temperature of $230^{\circ} \mathrm{C}$, an electron ionization ${ }^{+}$ion source, energy of $70 \mathrm{ev}$, detector voltage of $280 \mathrm{ev}$ and mass spectrometry detection range of 43.00-550.00.

$M w$ determination. Mws were calculated using a calibration curve obtained from various standard dextrans of different Mws (including Dextran Blue, Dextran T10, T40, T70, T110, T500 and glucose) (16).

UPPS-B1 (2 mg) or S-UPPS-B1 (2 mg) was dissolved in distilled water $(1 \mathrm{ml})$ and passed through a filter (pore size, $0.22 \mu \mathrm{m}$ ). Polysaccharides were then added to a gel-filtration chromatographic column [the same as aforementioned (HPLC; purity determination)] that was maintained at $40^{\circ} \mathrm{C}$, eluted with distilled water at a flow rate of $0.8 \mathrm{ml} / \mathrm{min}$ and detected via RID. The sample retention time was recorded.

Anti-tumor experiment in tumor-bearing mice. Mice (4 weeks; $20 \pm 2 \mathrm{~g}$; females and males) were used for experimentation and maintained under standard laboratory conditions. The ascites of S180 tumor-bearing mice that had been inoculated with ascites for 7 days and had grown well were extracted under aseptic conditions, and diluted to a cell suspension with a concentration of $1 \times 10^{7}$ cells $/ \mathrm{ml}$ with normal saline. A tumor cell suspension of $0.2 \mathrm{ml}$ was subcutaneously injected into the right forelimb armpit of each mouse to create a solid tumor model.

After $24 \mathrm{~h}$, the mice were randomly divided into five groups of 10 mice. The negative control group was treated with an equal volume of $0.9 \%$ normal saline. U. pinnatifida and Astragalus are traditional Chinese medicines and their active components are polysaccharides. Furthermore, astragalus polysaccharide (APS) is a clinically effective anti-tumor drug, so it was selected as the positive control in the animal experiment. The positive control group was treated with APS [intraperitoneal (i.p.), $100 \mathrm{mg} / \mathrm{kg}$ per day]. Treatment groups received UPPS-B1 $(25,50$ or $100 \mathrm{mg} / \mathrm{kg}$; i.p.) and S-UPPS-B1 (25, 50 or $100 \mathrm{mg} / \mathrm{kg}$; i.p.), a maximum tolerated dose experiment was performed beforehand (data not shown). In tumor-bearing mice, if weight loss of $>20 \%$, tumor diameter of $>1.5 \mathrm{~cm}$ or tumor showed ulcers and necrosis occurred, the experiment was suspended and the animals were euthanized. Pentobarbital sodium ( $45 \mathrm{mg} / \mathrm{kg}$; i.p.) was administered prior to cervical dislocation and the percentage of animals lost due to these issues was $<5 \%$. On the 8th day following administration, mice were weighed and sacrificed via cervical dislocation. Subsequently, tumor masses were removed and weighed. Tumor inhibition rates were calculated using the following formula:

Tumor inhibition rate $=[$ (average tumor weight of the control group-average tumor weight of the treatment group)/average tumor weight of the control group] $\mathrm{x} 100 \%$.

Statistical analysis. Values are expressed as the mean \pm standard deviation. Differences between groups were assessed by one-way analysis of variance followed by Dunnett's post-hoc test. SPSS16.0 software (SPSS, Inc.) was used for all statistical analyses. P $<0.05$ was considered to indicate statistical significance.

\section{Results}

Chemical characterization of the homogeneous polysaccharides. The UPPS crude polysaccharide was obtained from U. pinnatifida dry powder $(100 \mathrm{~g})$, which was formed following water extraction, ethanol precipitation, deproteinization and further separated via DEAE-52 ion-exchange chromatography (Fig. 1) and Sephadex G-200 size-exclusion chromatography (Fig. 2). The fractions were collected and dialyzed, and impurities were removed, including pigments and free proteins. Samples were then freeze-dried. As a result, one major polysaccharide fraction (UPPS-B1) was isolated with a yield of $79.78 \%$. HPLC profiles indicated that the polysaccharides were homogeneous, as each polysaccharide exhibited a symmetrical peak (Fig. 3).

Preliminary characterization of UPPS. The linear regression equation of the glucose standard curve was $\mathrm{A}=0.0371 \mathrm{C}$ $(\mu \mathrm{g} / \mathrm{ml})-0.005\left(\mathrm{R}^{2}=0.9986\right)$ and the content of glucose exhibited a positive linear correlation in the range of $0-25 \mu \mathrm{g} / \mathrm{ml}$. The linear regression equation of the uronic acid standard curve was $A=0.0049 C-0.005\left(R^{2}=0.9996\right)$ and the content of D-glucuronic acid demonstrated a positive linear correlation in the range of $0-100 \mu \mathrm{g} / \mathrm{ml}$. In addition, the linear regression 


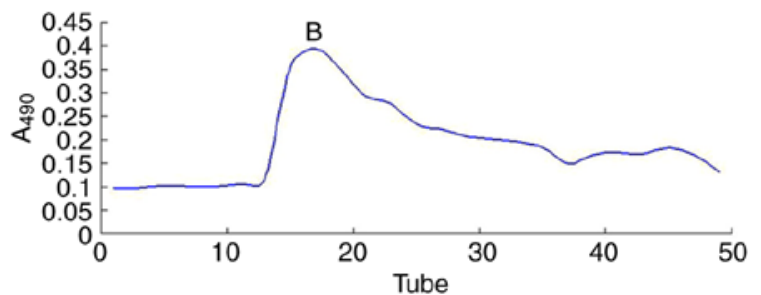

Figure 1. Elution curve of Undaria pinnatifida polysaccharides fraction-B1 on DEAE-52 eluted by $0.1 \mathrm{M} \mathrm{NaCl}$. $\mathrm{A}_{490}$, absorbance at $490 \mathrm{~nm}$.

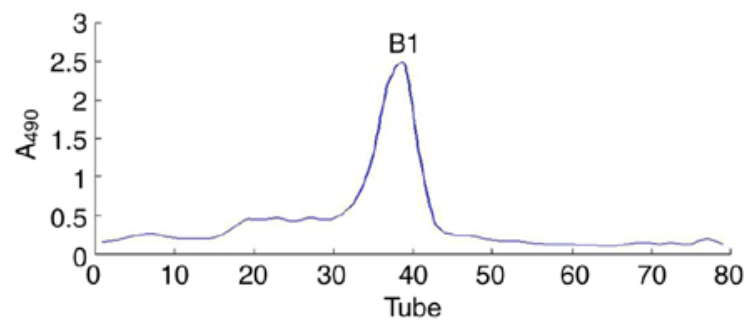

Figure 2. Elution curve of Undaria pinnatifida polysaccharides fraction-B1 on Sephadex G-200 eluted by distilled water. $\mathrm{A}_{490}$, absorbance at $490 \mathrm{~nm}$.

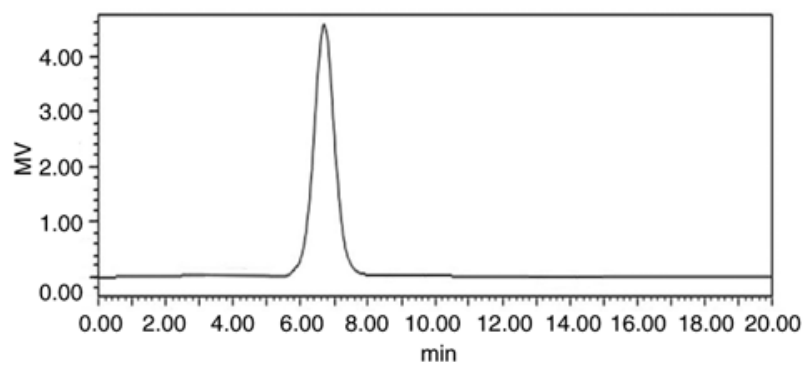

Figure 3. High-performance liquid chromatogram of Undaria pinnatifida polysaccharides fraction-B1. The ordinate of a liquid chromatography diagram is an electrical signal, usually expressed in MV (millivolts).

equation of the sulfate standard curve was $\mathrm{A}=0.0173 \mathrm{C}-0.0025$ $\left(\mathrm{R}^{2}=0.9994\right)$ and the content of $\mathrm{SO}_{4}{ }^{2-}$ exhibited a positive linear correlation in the range of $0-24 \mu \mathrm{g} / \mathrm{ml}$.

The results of the analysis indicated that the content of total sugar, sulfate radicals and uronic acid in UPPS-B1 were 79.78, 8.53 and $9.29 \%$, respectively. In UPPS-B1, acidic polysaccharides were identified. The results of the analysis indicated that the content of total sugar, sulfate radicals and uronic acid in S-UPPS-B1 was 77.28, 29.12 and 7.98\%, respectively. The substitution degree was 0.67 .

UV analysis. The UV spectra are presented in Fig. 4. There was no absorption peak at $260-280 \mathrm{~nm}$, indicating that the UPPS-B1 and S-UPPS-B1 did not include any nucleic acid or protein (17).

Fourier-transform IR analysis. The Fourier-transform IR spectra and analysis of the peaks are presented in Fig. 5 and Table I, respectively. The IR spectrum of UPPS-B1 and S-UPPS-B1 respectively exhibited a broad, stretched and intense characteristic band at $23,408.89$ and $3,442.03 \mathrm{~cm}^{-1}$ for the O-H group. Furthermore, a weak $\mathrm{C}-\mathrm{H}$ bond was
Table I. Functional groups of UPPS-B1 and S-UPPS-B1 in the infrared spectrum.

\begin{tabular}{lcc}
\hline & \multicolumn{2}{c}{ Absorption peak $\left(\mathrm{cm}^{-1}\right)$} \\
\cline { 2 - 3 } Vibration mode & UPPS-B1 & S-UPPS-B1 \\
\hline Stretching of O-H & 3408.89 & 3442.03 \\
Stretching of C-H & 2927.82 & 2926.84 \\
Asymmetric stretching of C=O & 1606.86 & 1638.62 \\
Symmetric stretching of C=O & 1420.29 & 1401.57 \\
Stretching of S=O & - & 1238.60 \\
Stretching of C-O-S & 822.81 & 822.29 \\
\hline
\end{tabular}

S-UPPS-B1, sulfated Undaria pinnatifida polysaccharides fraction B1.
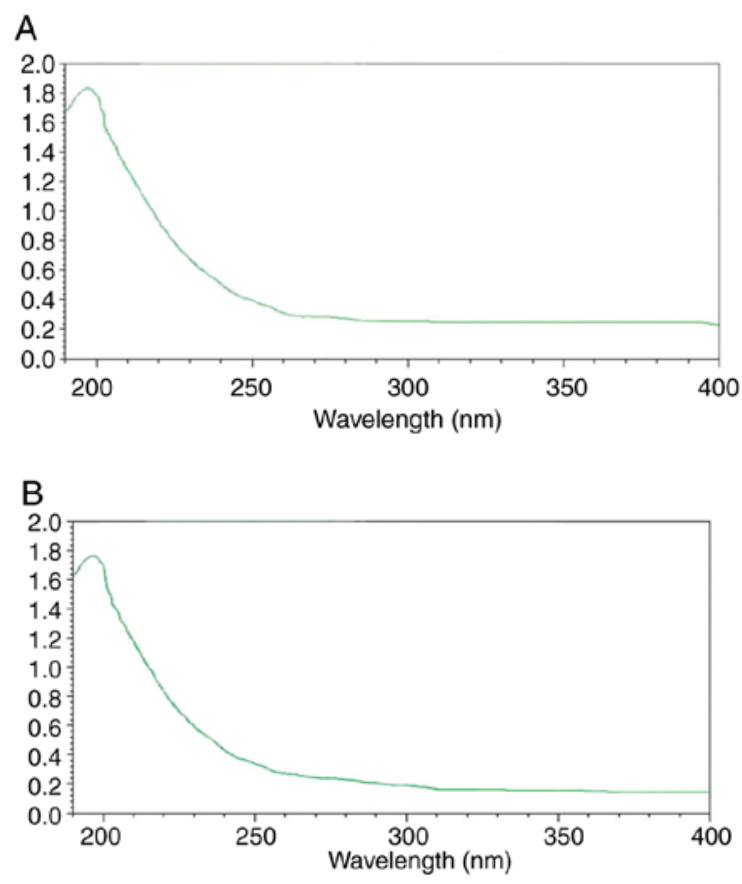

Figure 4. UV-visible light spectra of (A) UPPS-B1 and (B) sulfated UPPS-B1. The absorption value is indicated by ' $\mathrm{A}$ ' on the UV detector, A unit is 1 . UV, ultraviolet; UPPS-B1, Undaria pinnatifida polysaccharides fraction B1.

represented by the bands at $\sim 2,927.82$ and $2,926.84 \mathrm{~cm}^{-1}$, respectively. The $\mathrm{O}-\mathrm{H}$ and $\mathrm{C}-\mathrm{H}$ absorption bands were typical absorptions of polysaccharides (18). The characteristic absorption peaks of $-\mathrm{O}-\mathrm{SO}_{3}$ were present at $\sim 1,238$ and $822 \mathrm{~cm}^{-1}$. In addition, $-\mathrm{COOH}$ was represented by bands at $\sim 1,606$ and $1,420 \mathrm{~cm}^{-1}$. A strong absorption was also observed in the region of 1,000-1,200 $\mathrm{cm}^{-1}$, which was attributed to C-O-C and $\mathrm{C}-\mathrm{OH}$ bands. Other absorption peaks of pyranose were present at $\sim 1,037.08$ and $1,046.08 \mathrm{~cm}^{-1}$. A peak at $887.27 \mathrm{~cm}^{-1}$ indicated that the glycosidic bonds in UPPS-B1 were of the $\beta$-type. A peak at $856.54 \mathrm{~cm}^{-1}$ indicated that the glycosidic bonds in S-UPPS-B1 were of the $\alpha$-type.

Analysis of the monosaccharide composition of polysaccharides. The monosaccharide composition of each polysaccharide 

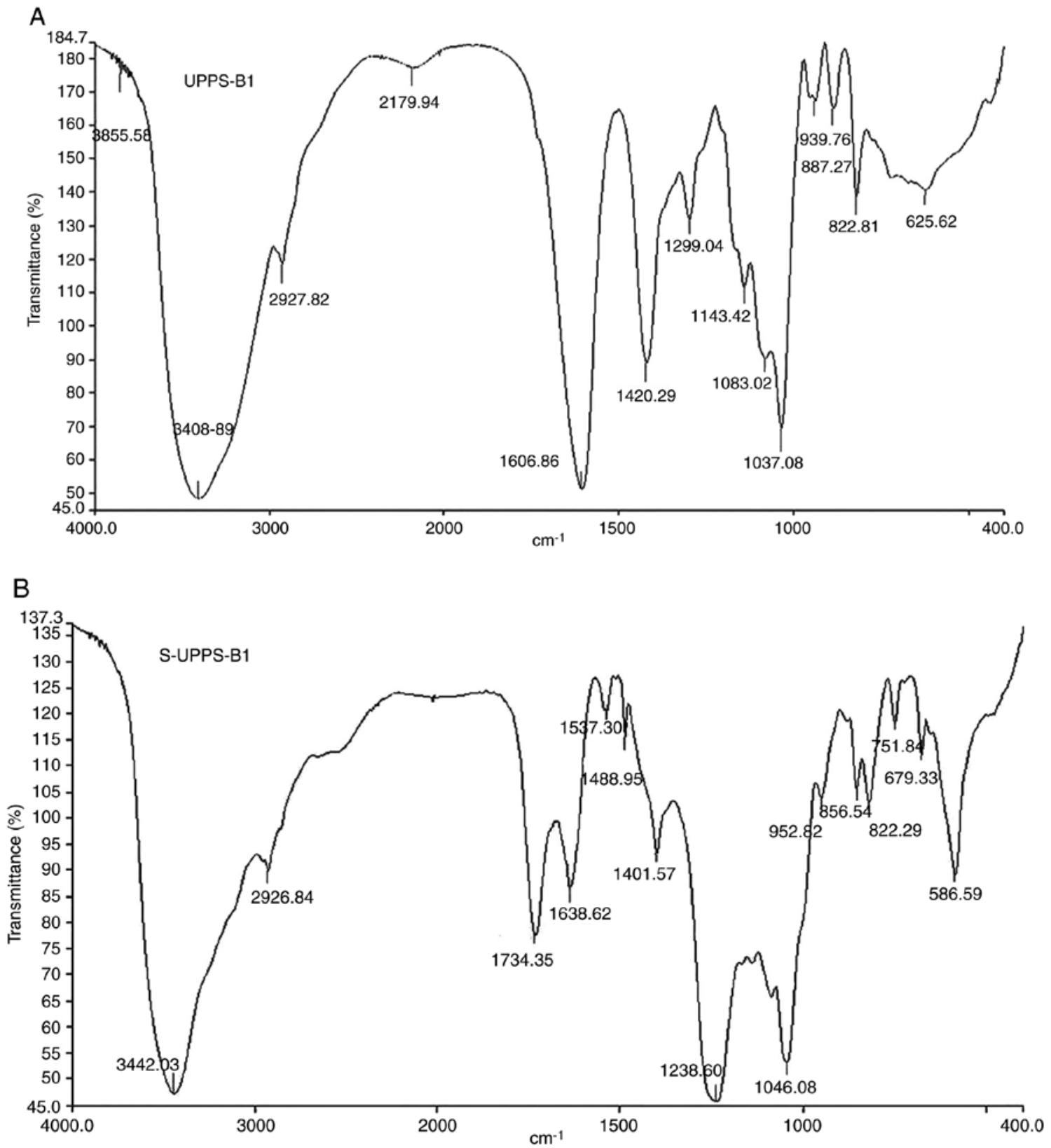

Figure 5. Fourier-transformed infrared spectra of (A) UPPS-B1 and (B) sulfated UPPS-B1. UPPS-B1, Undaria pinnatifida polysaccharides fraction B1.

was determined using GC-MS (Table II). The results revealed that UPPS-B1 and S-UPPS-B1 exhibited a different monosaccharide composition, indicating that their individual features may be relevant to their biological activities.

$M w$. The linear regression equation of the Mw standard curve was calculated as $\operatorname{lgMw}=-6.7881 \mathrm{~T}_{\mathrm{R}}$ (retention time) +8.7134 $\left(r^{2}=0.9984\right)$. Furthermore, the average Mws of UPPS-B1 and S-UPPS-B1 were calculated as 37 and $110 \mathrm{kDa}$, respectively.

Tumor growth inhibition. The results of the tumor growth inhibition experiment are presented in Table III. Compared with the control, tumor inhibition rates in the high, medium and low-dose UPPS-B1-treated mice were 54.77, 36.96 and $20.83 \%$, respectively. Furthermore, the tumor inhibition rates in the high, middle and low-dose S-UPPS-B1-treated groups were $63.05,50.41$ and $39.52 \%$, respectively. The tumor inhibition rate of APS was 54.01\%. The S-UPPS-B1 group demonstrated a significant reduction in tumor weight compared with the control group.

\section{Discussion}

The major polysaccharide fraction from U. Pinnatifida, UPPS-B1, was purified by DEAE-52 and Sephadex G-200 column chromatography. The chlorosulfonic acid-pyridine method was applied for sulfation modification. Subsequently, UPPS-B1 and the sulfated derivative, S-UPPS-B1, were characterized using chemical analysis, UV-visible spectroscopy, FT-IR spectroscopy, gas chromatography and HPLC. The Mw and monosaccharide composition of polysaccharides was determined. The in vivo study then indicated that UPPS-B1 and S-UPPS-B1 exhibited marked anti-tumor activity. 
Table II. Monosaccharide composition of polysaccharides isolated from UPPS.

\begin{tabular}{lccrr}
\hline Substance & Xylose $(\%)$ & Mannose $(\%)$ & Glucose $(\%)$ & Galactose $(\%)$ \\
\hline UPPS-B1 & 7.946 & 8.719 & 11.551 & 9.773 \\
S-UPPS-B1 & 1.000 & 9.700 & 6.400 & 1.600 \\
\hline
\end{tabular}

S-UPPS-B1, sulfated Undaria pinnatifida polysaccharides fraction B1.

Table III. Inhibitory effect of UPPS-B1 and S-UPPS-B1 on solid S180 tumors in mice.

\begin{tabular}{lcc}
\hline Group & Dose $[\mathrm{mg} /(\mathrm{kg} \cdot$ day $)]$ & Tumor weight $(\mathrm{g})$ \\
\hline Control & - & $1.0755 \pm 0.1075$ \\
APS & 100 & $0.4946 \pm 0.0609^{\mathrm{a}}$ \\
UPPS-B1 & 25 & $0.8515 \pm 0.0215^{\mathrm{a}}$ \\
& 50 & $0.6780 \pm 0.0640^{\mathrm{a}}$ \\
& 100 & $0.4864 \pm 0.0479^{\mathrm{a}}$ \\
S-UPPS-B1 & 25 & $0.6505 \pm 0.0714^{\mathrm{a}}$ \\
& 50 & $0.5333 \pm 0.1354^{\mathrm{a}}$ \\
& 100 & $0.3974 \pm 0.0465^{\mathrm{a}}$ \\
\hline
\end{tabular}

${ }^{\mathrm{a}} \mathrm{P}<0.05$ compared with the normal control. Values are expressed as the mean \pm standard deviation $(\mathrm{n}=10)$. S-UPPS-B1, sulfated Undaria pinnatifida polysaccharides fraction B1; APS, Astragalus polysaccharides.

Malignant tumors are increasingly posing a threat to human health. The search for highly efficient anti-neoplastic drugs with low toxicity have therefore become the focus of cancer treatment. Natural compounds are effective therapeutic agents and numerous polysaccharides exert anti-tumor effects. The biological activity of polysaccharides is closely associated with their structure and physicochemical properties. The steric and electrostatic repulsion effects of sulfate groups may alter the spatial structure of polysaccharides and the flexion of the sugar chain, thus increasing water solubility and resulting in changes to their biological activity $(19,20)$.

After sulfated modification, the contents of xylose, glucose and galactose were markedly decreased in the sulfated derivatives examined in the present study, while there was a slight increase in the content of mannose. These results were consistent with those of a previous study (16), in which sulfated modifications caused alterations in the monosaccharide composition of polysaccharides. However, in the present study, the application of sulfated modification did not result in the destruction of the major chain of the sulfated derivatives.

The Mw of sulfated polysaccharides is an important parameter that affects the biological activity of polysaccharides (21). In general, the degradation of polysaccharides is accomplished during the sulfation reaction via the chlorosulfonic acid method (22). However, the results of the present study revealed that $S$-UPPS-B1 exhibited an increase in Mw compared with that of UPPS-B1, indicating that these sulfated derivatives were successfully produced in the present study without degradation.

Nuclear magnetic resonance (NMR) may provide a vast amount of information on the monosaccharide composition of polysaccharides, the sequence of monosaccharide residues, the position of monosaccharide residues in glycoside bonds, the type of ring structure and the configuration of glycoside bonds. Therefore, in order to obtain comprehensive information on the structure of polysaccharides, further research by our group will endeavor to use NMR technology to assign ${ }^{1} \mathrm{H}$ and ${ }^{13} \mathrm{C}$ signals of each sugar residue in UPPS-B1 and S-UPPS-B1.

The results of the anti-tumor experiment revealed that, after sulfated modification, the anti-tumor activity of S-UPPS-B1 was greater than that of UPPS-B1 at the same dose. MTT assay has been done by other group as a preliminary experiment. This may be due to the sulfated modification altering the molecular structure and sulfate content of the polysaccharide, leading to changes in biological activity.

The crude polysaccharide obtained from $U$. pinnatifida in the present study predominantly contained the water-extractable polysaccharide UPPS-B1, which was purified via DEAE-52 and Sephadex G-200 chromatography. S-UPPS-B1, with a substitution degree of 0.67 , was synthesized using the chlorosulfonic acid-pyridine method. The HPLC results indicated that UPPS-B1 and S-UPPS-B1 were homogeneous and the compounds had an average Mw of 37 and $110 \mathrm{kDa}$, respectively. The GC-MS indicated that UPPS-B1 and S-UPPS-B1 were primarily composed of xylose, mannose, glucose, galactose and glucuronic acid. The results indicated that sulfation of polysaccharides may improve solubility and result in high anti-tumor activity. S-UPPS that was synthesized in the present study exhibited enhanced anti-tumor activity when compared with that of UPPS. However, the structural properties and degree of substitution, as well as the underlying anti-tumor mechanisms, require further study. The present study provides a theoretical basis for the structure-activity association of polysaccharides, which may lead to pharmaceutical of the U. pinnatifida polysaccharides.

\section{Acknowledgements}

In the present study, separation and purification of polysaccharides from U. Pinnatifida polysaccharides was performed using the alcohol precipitation method, sulfation was performed using the chlorsulfonate-pyridine method and UV and IR spectrum analyses were used for verification. The subsequent in vivo study indicated that UPPS and S-UPPS exhibited anti-tumor activity. This is a previously published conference abstract (23). 


\section{Funding}

This work was supported by the Harbin Municipal Science and Technology Bureau Project (grant nos. 2016RQQXJ124 and 2016RAXXJ064), the Innovation Talent Project of the Education Department of Heilongjiang Province (grant no. UNPYSCT-2016181) and the Science Foundation Project of Harbin University of Commerce (grant no. 18XN067).

\section{Availability of data and materials}

The datasets used and/or analyzed during the current study are available from the corresponding author on reasonable request.

\section{Authors' contributions}

YBJ designed the study, FLW performed the research and wrote the manuscript. YBJ, FLW and BY analyzed the data and revised the manuscript.

\section{Ethics approval and consent to participate}

The experimental protocol was approved by the Institutional Animal Care and Use Committee of Heilongjiang University of Chinese Medicine (Harbin, China).

\section{Patient consent for publication}

Not applicable.

\section{Competing interests}

The authors declare that they have no competing interests.

\section{References}

1. Costa LS, Fidelis GP, Cordeiro SL, Oliveira RM, Sabry DA, Câmara RB, Nobre LT, Costa MS, Almeida-Lima J, Farias EH, et al: Biological activities of sulfated polysaccharides from tropical seaweeds. Biomed Pharmacother 64: 21-28, 2010.

2. Zhou AY, Robertson J, Hamid N, Ma Q and Lu J: Changes in total nitrogen and amino acid composition of New Zealand Undaria pinnatifida with growth, location and plant parts. Food Chem 186 319-325, 2015.

3. Kim KJ, Yoon KY and Lee BY: Low molecular weight fucoidan from the sporophyll of Undaria pinnatifida suppresses inflammation by promoting the inhibition of mitogen-activated protein kinases and oxidative stress in RAW264.7 cells. Fitoterapia 83 : 1628-1635, 2012.

4. Vishchuk OS, Ermakova SP and Zwaqintseva TN: The fucoidans from brown algae of far-eastern seas: Anti-tumor activity and structure-function relationship. Food Chem 141: 1211-1217, 2013.

5. Wang JL, Guo HY, Zhang J, Wang XF, Zhao B, Yao J and Wang Y: Sulfated modification, characterization and structure-antioxidant relationships of Artemisia sphaerocephala polysaccharides. Carbohydr Polym 81: 897-905, 2010.
6. Sun ZW, He YL, Liang ZH, Zhou WW and Niu TG: Sulfation of $(1 \rightarrow 3)-\beta$-D-glucan from the fruiting bodies of Russula virescens and antitumor activities of the modifiers. Carbohydr Polym 77: 628-633, 2009

7. Wang J,Hu Y, Wang D, Liu J,Zhang J, Abula S, Zhao B and Ruan S: Sulfated modification can enhance the immune-enhancing activity of lycium barbarum polysaccharides. Cell Immunol 263: 219-223, 2010.

8. Wang Y, Hu X, Han J, Ni L, Tang X, Hu Y and Chen T: Integrated method of thermosensitive triblock copolymer-salt aqueous two phase extraction and dialysis membrane separation for purification of lyceum barbarum polysaccharide. Food Chem 194: 257-624, 2016.

9. Wang ZJ, Luo DH and Ena C: Optimization of polysaccharides extraction from gynostemma pentaphyllum makino using uniform design. Carbohydr Polym 69: 311-317, 2007.

10. Blumenkrantz N and Asboe-Hansen G: New method for quantitative determination of uronic acids. Anal Biochem 54: 484-489, 1973.

11. Ji CF, Ji YB and Meng DY: Sulfated modification and anti-tumor activity of laminarin. Exp Ther Med 6: 1259-1264, 2013.

12. Yin X, You Q, Jiang Z and Zhou X: Optimization for ultrasonic-microwave synergistic extraction of polysaccharides from cornus officinalis and characterization of polysaccharides. Int J Biol Macromol 83: 226-232, 2016.

13. Lü H, Gao Y, Shan H and Lin Y: Preparation and antibacterial activity studies of degraded polysaccharide selenide from Enteromorpha prolifera. Carbohydr Polym 107: 98-102, 2014.

14. Zhang TT, Lu CL, Jiang JG, Wang M, Wang DM and Zhu W: Bioactivities and extraction optimization of crude polysaccharides from the fruits and leaves of Rubus chingii hu. Carbohydr Polym 130: 307-315, 2015.

15. Ji X, Peng Q, Yuan Y, Shen J, Xie X and Wang M: Isolation, structures and bioactivities of the polysaccharides from jujube fruit (Ziziphus jujube Mill.): A review. Food Chem 227: 349-357, 2017.

16. Wang ZJ, Luo DH and Liang ZY: Structure of polysaccharides from the fruiting body of Hericium erinaceus Pers. Carbohydr Polym 57: 241-247, 2004.

17. Yu XH, Liu Y, Wu XL, Liu LZ, Fu W and Song DD: Isolation, purification, characterization and immunostimulatory activity of polysaccharides derived from American ginseng. Carbohydr Polym 156: 9-18, 2017.

18. Yi P, Li N, Wan JB, Zhang D, Li M and Yan C: Structural characterization and antioxidant activity of a heteropolysaccharide from ganoderma capense. Carbohydr Polym 121: 183-189, 2015.

19. Bao H, Choi WS and You S: Effect of sulfated modification on the molecular characteristics and biological activities of polysaccharides from Hypsizigus marmoreus. Biosci Biotechnol Biochem 74: 1408-1414, 2010.

20. Zhang Y, Lu X, Zhang Y, Qin L and Zhang J: Sulfated modification and immunomodulatory activity of water-soluble polysaccharides derived from fresh Chinese persimmon fruit. Int J Biol Macromol 46: 67-71, 2010.

21. Xie JH, Wang ZJ, Shen MY, Nie SP, Gong B, Li HS, Zhao Q, Li WJ and Xie MY: Sulfated modification, characterization and antioxidant activities of polysaccharide from Cyclocarya paliurus. Food Hydrocolloids 53: 7-15, 2016.

22. Chen Y, Zhang H, Wang Y, Nie S, Li C and Xie M: Sulfated modification of the polysaccharides from Ganoderma atrum, and their antioxidant and immunomodulating activities. Food Chem 186: 231-238, 2015.

23. Wang FL, Yang B and Ji YB: Purification of polysaccharides from Undaria pinnatifida and antitumor effect of sulfated before and after modification[C]. Kunming: The 10th National Conference on Medicinal Plants and Phytomedicines, 113, 2011. 